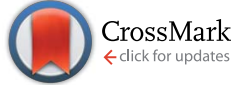

Cite this: Soft Matter, 2015, 11, 1158

Received 26th May 2014

Accepted 17th December 2014

DOI: $10.1039 / c 4 s m 01142 b$

www.rsc.org/softmatter

\section{Methods to determine the pressure dependence of the molecular order parameter in (bio) macromolecular fibres $\uparrow$}

\begin{abstract}
Arthur Markus Anton, ${ }^{* a}$ Christof Gutsche, ${ }^{\text {ab }}$ Wilhelm Kossack ${ }^{a}$ and Friedrich Kremer $^{a}$
The experimental realization and an algorithm for analysing the pressure dependence of the molecular order parameter of specific structural moieties in (bio)macromolecular fibres are described. By employing a diamond anvil cell (DAC) the polarization-dependent IR-transmission and in parallel, using an integrated microscope, the macroscopic orientation of the fibres is determined. This enables one to separate between order and disorder at macroscopic and microscopic scales. Using the example of spider silk the pressure dependence of the molecular order parameter of alanine groups being located within nano-crystalline building blocks is deduced and found to decrease reversibly by $0.01 \mathrm{GPa}^{-1}$ when varying the external hydrostatic pressure between 0 and $3 \mathrm{GPa}$.
\end{abstract}

\section{Introduction}

Orientation on the molecular scale plays a fundamental role in materials science, for example anisotropic mechanical or optical properties. ${ }^{1-3}$ This information can be obtained by performing orientation-dependent measurements in combination with externally applied mechanical fields as uniaxial stress or hydrostatic pressure. ${ }^{4-6}$ In case of the latter, high pressure experiments in spectroscopy are performed routinely, wherefore a diamond anvil cell (DAC) is commonly employed..$^{7-10}$ Due to its geometrical constraints, resulting from two diamonds pressing against a gasket containing the sample within a small hole $(<\mathrm{mm})$, which itself undergoes deformation during pressure application, performing polarization-dependent studies on a macroscopically oriented sample is a challenging task. Materials, that ensure the alignment of the sample, may affect the measurement by their own (background) signals, and furthermore could hamper hydrostaticity due to their mechanical properties. Additionally, access to the sample compartment from outside is impossible after a first application of pressure. Due to that reasons, Colomban et al. used one thick fibre cut properly to fit into the gasket's bore instead of many thin ones

${ }^{a}$ Universität Leipzig, Fakultät für Physik und Geowissenschaften, Institut für Experimentelle Physik I, Abteilung Molekülphysik, Linnéstraße 5, D-04103 Leipzig, Germany.E-mail: anton@physik.uni-leipzig.de

${ }^{b}$ Universität Leipzig, Medizinische Fakultät, Poliklinik für Zahnerhaltung und Parodontologie, Liebigstraße 12, D-04103 Leipzig, Germany

$\dagger$ Electronic supplementary information (ESI) available: The definition of the molecular order parameter in general and in case of rotation symmetry (S1), the conversion between the molecular order parameter and the dichroic ratio (S2), the convolution of a Gaussian distribution function with a discrete distribution function (S3), and the convolution of two Gaussian functions (S4), as well as, eqn S1 and S2. See DOI: 10.1039/c4sm01142b to perform polarized Raman spectroscopy. ${ }^{11}$ Unfortunately, preparing a thicker, and hence less flexible, sample is not possible in any cases. Recently, Ene et al. published X-ray measurements on spider silk, wherefore these highly flexible fibres exhibiting a diameter of 3-5 $\mu \mathrm{m}$ have to be aligned. ${ }^{12}$ For that, they used a home build pressure cell employing water as pressure transmitting medium being limited to $0.7 \mathrm{GPa}^{13}$ However, methods enabling measurements on aligned highly flexible fibres at elevated or high hydrostatic pressures are rare. In the following, we present two novel and easy to use approaches to transfer gratings of aligned fibres into a DAC. Major ampullate spider silk fibres are employed, because its inherent high anisotropy of a $\beta$-sheet specific polyalanine vibration $^{5}$ and the material's great flexibility, emphasize these fibres to an excellent model system probing geometrical misalignment and evaluating a method's feasibility avoiding geometrical distortions. The grating's deformation upon the application of pressure is detected through measuring the apparent dichroic ratio of a specific IR absorption band, as well as, using optical microscopy. With the help of the latter we separate the influence of the macroscopic deformation of the fibre grating from the pressure-dependent microscopic distribution of transition moments, both having an effect on the IR measurement.

\section{Experimental}

Stainless steel gaskets ( 250 or $300 \mu \mathrm{m}$ thick) and synthetic type 2a diamonds with a culet size of $1.0 \mathrm{~mm}$ are employed. The gasket's pre-indentation is kept at a minimum to overcome any distortion of the grid; just enough to estimate the diamonds' contact area. According to the culet size, a hole with a diameter of $0.5 \mathrm{~mm}$ is drilled into the gasket at the middle of the 
diamonds' contact area. The spider silk samples are made from major ampullate (drageline) silk of the spider Nephila edulis using the method of forced silking. ${ }^{5}$ The spiders are reared on a diet of crickets.

To produce a grating of aligned fibres the following two techniques are used: (A) for one approach a single thread is reeled around two parallel rods. ${ }^{5}$ Afterwards, one side of the so produced double-sided grating is glued directly on the gasket's surface covering its bore (Method A, Fig. 1a). For that, twocomponent glue is employed, because this adhesive provides high mechanical resistance while avoiding the effect of solvents. The free standing side of the double-sided grating is removed, enabling to mount the sample into the DAC and eliminating additional absorption and scattering. Please note that the term grating is chosen in analogy to optical grating and does not represent a crossed arrangement. Instead, the fibres are aligned in a parallel fashion since its displacement is controlled by a computer-operated stage.

(B) An other approach to produce a grating of aligned fibres is winding the thread directly around the gasket covering its hole (Method B, Fig. 1b). For that reason, the edges of the initially circular-shaped gasket are flattened. Nail polish is used to fix the fibres on one side of the gasket before cutting off the other side of the grating. This type of glue is employed, because it exhibits excellent wettability. A gap of at least $1 \mathrm{~mm}$ is kept between contact site and sample compartment. In addition, alternative approaches requiring enhanced technological efforts compared to the two afore-mentioned ones are provided in the ESI. $\dagger$

After the hole within the gasket is covered with a grating of aligned spider silk fibres, paraffin oil is added which reduces light scattering of the fibres without affecting their mechanical properties ${ }^{5}$ and also acts as pressure transmitting medium. ${ }^{6,14}$ Hydrostatic pressure is applied by means of a

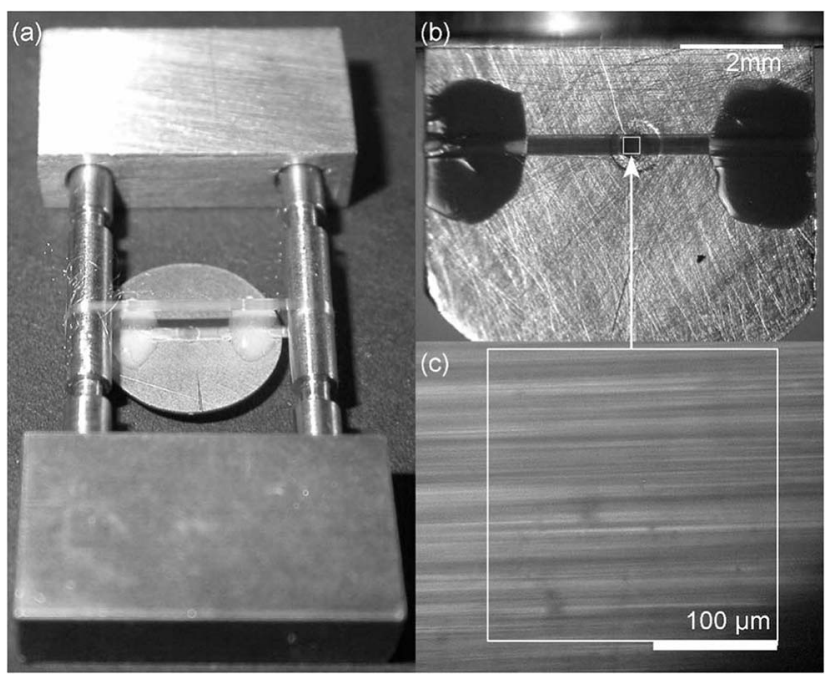

Fig. 1 A grating of spider silk fibres wound around two stainless steel rods and glued on a gasket (a). Alternatively, a thread is directly wound around a gasket with flattened edges (b). Both methods are convenient to produce a grating of aligned fibres as evident in the micrograph (c). $\mu$ ScopeDAC-HT(G) diamond anvil cell (easyLab) and determined in situ from the pressure-dependent shift of the ruby $\mathrm{R}_{1}$ fluorescence line. ${ }^{15}$ Infrared spectra are recorded in transmission mode on a Fourier-transform-infrared (FTIR)-spectrometer (FTS 6000, BioRad) equipped with an IR-microscope (UMA-500, BioRad) and a mercury-cadmium-telluride (MCT) detector (Kolmar Technologies) using a spectral resolution of 4 $\mathrm{cm}^{-1}$. The IR-light is linearly polarized before entering the DAC (for details regarding the setup see the ref. 5 and 6).

The difference of the vibrational absorption at $\bar{\nu}=965 \mathrm{~cm}^{-1}$ depending on the IR light's polarization, typical for major ampullate spider silk, is utilized to determine the dichroic ration ( $c f$. eqn (2) and molecular order parameter ESI S2) of the $\beta$-sheet polyalanine nanocrystals embedded in an glycine-rich amorphous, glassy matrix. ${ }^{5,16}$ As long as one employs a grating of parallel fibres the dichroic ratio of this arrangement is identical to that of a single fibre and one is able to extract information of the microscopic orientation of the IR transition moments. ${ }^{1,5}$ In case the spider silk grating is deformed and the fibres' alignment is distorted, the outcome of the IR measurement depends on the microscopic orientation of the IR transition moments, as before, but also on the macroscopic geometrical orientation of the fibres. To evaluate the latter and to separate its influence on the IR measurements from the pressure-dependent microscopic orientation, we make use of optical microscopy as independent technique complimentary to IR spectroscopy.

\section{Results and discussion}

After producing a grating of aligned fibres and transferring it into the DAC, the sample and the gasket are deformed as the pressure is applied for the first time resulting in a loss of the fibres' alignment (Fig. 2). Despite a clearly visible deterioration, certain areas with fibres in parallel can still be found. On expense of signal-to-noise ratio and measurement time, the region of interest (ROI) of the focusing optics can be reduced resulting in a more-pronounced spectral dichroism and finally a larger order parameter. This argument is valid for both Method A and B described above.

Especially when using Method B, it is noteworthy that due to the insufficient pre-indentation, the gasket's bore expands with rising pressure, ${ }^{10,17}$ causing an elliptical shape of the hole. This deformation is a result of the gasket's asymmetric shape and affects the previously cut edges, because of a lack of material at these sites. At ( 2.0 or $2.7 \pm 0.2) \mathrm{GPa}$ (derived from the externally applied membrane pressure) the hole enlarges as wide as the pressure is not preserved anymore. Large expansion of the bore can lead to damage on the diamonds, ${ }^{10}$ however, in both of our experiments performed using Method $\mathrm{B}$, the diamonds remained intact.

For estimating the geometrical distribution of the fibres, a micrograph of the sample compartment is divided into equal square regions, where the fibres are in parallel and their orientation angle relative to a fixed direction can be estimated (Fig. 2). Afterwards, a histogram of orientation angles modelling the fibres arrangement within the image plane is compiled. The 

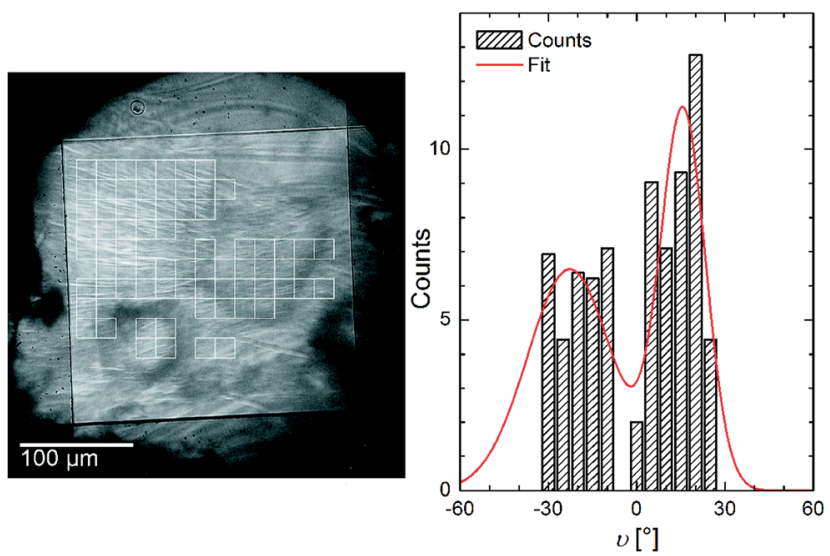

Fig. 2 A scheme illustrating how to determine the macroscopic, geometrical orientation of the spider silk fibres (here after subsequent pressure cycles). A micrograph is divided up into equally sized squares $(50 \times 50$ pixel) in which the orientation angle with respect to vertical orientation is estimated. The bright square indicates the $250 \times 250$ $\mu \mathrm{m}^{2} \mathrm{ROI}$ of the IR microscope. A histogram is compiled corrected for locating the mean at $0^{\circ}$, as automatically performed when applying eqn (1) on the measured spectra. The solid line indicates an optional fit assuming a sum of two Gaussian distribution functions. The obtained parameters can be found in Table 1.

heights of the histogram bars are corrected to overcome any deviation of the fibre length, and consequently the sample volume, within a square region depending on the orientation angle $\left(h=h_{0} / \cos (\theta)\right)$. Additionally, it is possible to fit a Gaussian function (or a sum of those) to the histogram enabling a continuous description of the fibres' distribution.

To specify the alignment of the sample fibres in a more refined way, polarization-dependent FTIR spectra are recorded and corrected for the (also pressure-dependent) paraffin absorption. For that a polarization-independent peak at 890 $\mathrm{cm}^{-1}$ is used as a measure for the sample's paraffin content. Especially in case of this sample arrangement, the volume of the pressure transmitting medium is significantly higher than for other measurements $(\approx 100 \times$ compared to former experiments $\left.{ }^{5,6}\right)$, resulting in an enhanced background signal. As a consequence of refractive index mismatch and birefringent properties of spider silk, clear interference patterns are evident in the paraffin background spectra which has to be reassessed, while those are absent in case of the spider silk measurements. After subtraction of a linear baseline from the paraffin-corrected spectra, the well separated polyalanine peak is modelled with a pseudo-Voigt function, as proposed by Stancik and Brauns. ${ }^{18}$ Afterwards, the maximum absorbance $A_{\max }$ appearing at a polarization angle $\Omega$ is determined through fitting eqn (1) to the data:

$$
A(\phi)=-\log _{10}\left[10^{-A_{\max }} \cos ^{2}(\phi-\Omega)+10^{-A_{\min }} \sin ^{2}(\phi-\Omega)\right],
$$

where $\phi$ represents the polarization angle and $A_{\min }$ the absorbance value when the light is polarized perpendicular to $\Omega .^{5}$ Subsequently, these measures are used to calculate the apparent dichroic ratio

$$
D=\frac{A_{\max }}{A_{\min }}
$$

Note that we are focusing on the dichroic ratio as physical quantity instead of the order parameter, because to calculate the latter all three values of the absorbance strength relative to the principle axes has to be known, but only two of them can be measured directly when the sample is inside the DAC. Consequently, assumptions has to be made to work out the molecular order parameter based on the measured dichroic ratio, which are well justified in case of rotational symmetric fibres (see ESI S1†).

In case the spider silk fibres are aligned and form a grating (reeled around two parallel rods) a dichroic ratio of $D_{\mathrm{A}}^{\mathrm{ref}}=$ $22.4 \pm 0.5$ or $D_{\mathrm{B}}^{\mathrm{ref}}=20.1 \pm 0.5\left(S_{\mathrm{A}}^{\mathrm{ref}}=0.88 \pm 0.01, S_{\mathrm{B}}^{\text {ref }}=0.86 \pm\right.$ 0.01 ) can be found (Fig. 3a). In case the sample is prepared using Method A, a deteriorated dichroism becomes evident (Fig. $3 \mathrm{~b}$ ) and the dichroic ratio decreases to $D_{\mathrm{A}}=$ $5.5 \pm 1.1$ when closing the pressure cell, due to the sample grating's misalignment. A measurement uncertainty for fitting the integrated absorbance of $5 \%$ is estimated, resulting in a total uncertainty of $20 \%$ for the dichroic ratio of samples under load. The reference samples are not concerned here. During the application of hydrostatic pressure $D_{\mathrm{A}}$ is reduced further, but the initial value is restored during relaxation without any indication of hysteresis or persistent deterioration (Fig. 3g). Performing a second measurement cycle might detect additional plastic deformations, which are absent in our study.

In case of Method B the dichroic ratio appears to be slightly reduced when the thread is wound directly around the gasket $\left(D_{\mathrm{B}}^{\mathrm{ref}}=17.3 \pm 0.5, S_{\mathrm{B}}^{\mathrm{ref}}=0.84 \pm 0.02\right)$ compared to winding around rods (see above). This reduction is caused by exchanging rods exhibiting a circular cross section, where the thread can cling to their smooth surface, by a flattened, $300 \mu \mathrm{m}$ thick gasket with sharp edges. Despite the fact that the hole of the gasket has a diameter of $500 \mu \mathrm{m}$, whereas the microscope's ROI amounts to $250 \times 250 \mu \mathrm{m}^{2}$, it is not possible to excluded it as additional optical element deteriorating the measurement conditions. However, similar to Method A, $D_{\mathrm{B}}$ is reduced upon closing the cell due to geometrical distortions of the grating to $D_{\mathrm{B}}=6.0 \pm 1.2$, exhibiting a slightly larger value compared to $D_{\mathrm{A}}$. A possible explanation might be found in the sample preparation. In Method A the grating is glued on the gasket's surface resulting in a gap due to the adhesive, whereas in Method B the thread is in direct contact to the gasket's surface. This gab may cause a stronger misalignment, and hence, a lowered dichroism.

To estimate the reversible change of the dichroic ratio in the range from 0 to $2.98 \mathrm{GPa}$ a linear function is fitted to the measured values of $D$ which results in a slope of $a_{\mathrm{A}}=(-0.07 \pm$ $0.14) \mathrm{GPa}^{-1}$ and an intercept of $b_{\mathrm{A}}=3.74 \pm 0.23$. In that way one is able to separate the decrease of the apparent dichroic ratio, due to geometrical misalignment from closing the DAC, and its variation as a result of hydrostatic pressure. 

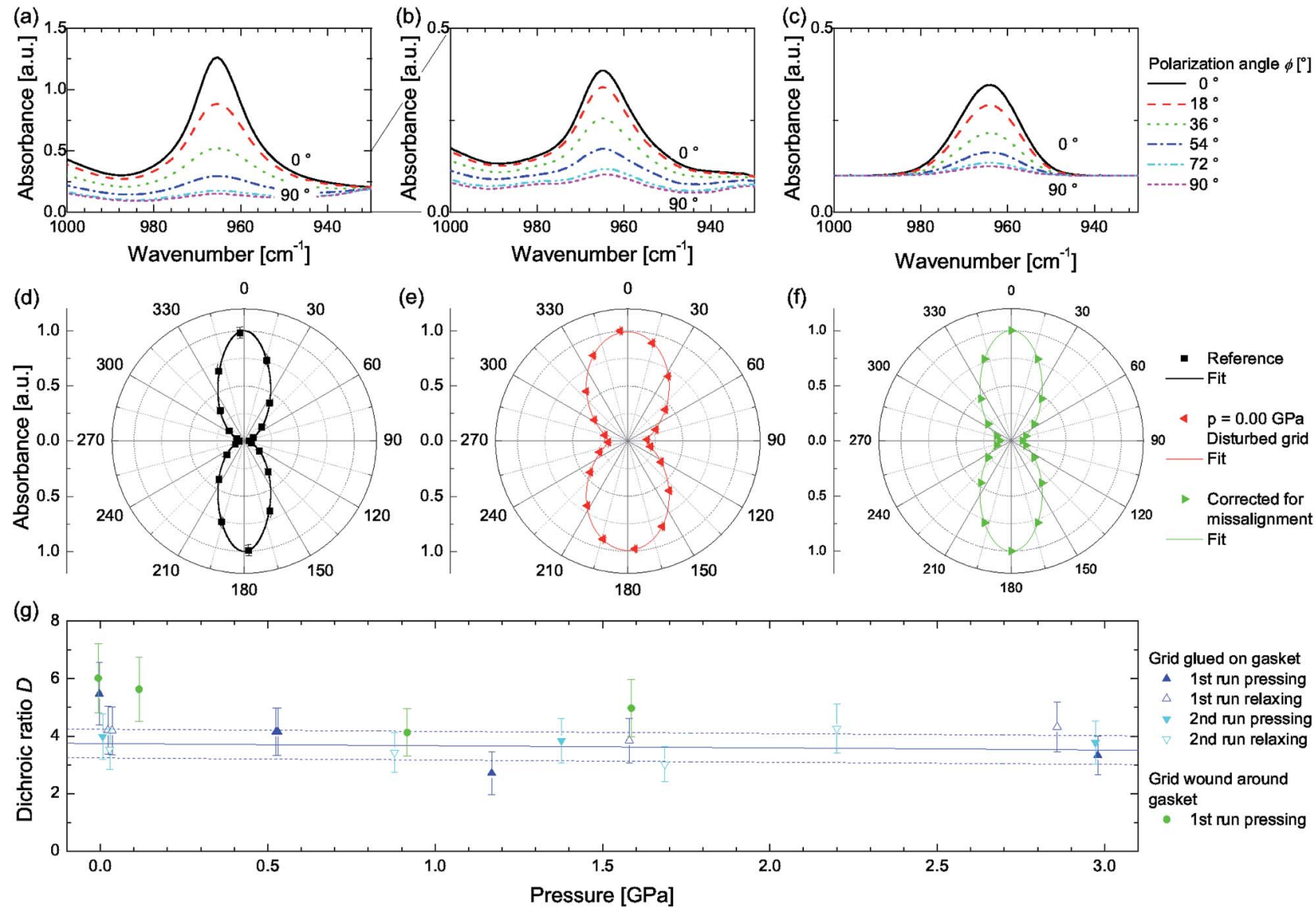

Fig. 3 Polarization-dependent spectra showing IR-dichroism of the band located at $965 \mathrm{~cm}^{-1}$ representing the alanine moiety (a) for a fresh, native reference sample (wound around rods), (b) after closing the DAC at $0.00 \mathrm{GPa}$, and (c) recalculated by correcting for the geometrical distortion of the grating. ( $d-f)$ Polar representation of (a)-(c) normalized to 1 along with fits according to eqn (1). (g) Apparent dichroic ratio $D$ depending on the applied hydrostatic pressure. The blue line represents a linear fit, while the dashed lines correspond to $D \pm 0.5$.

To model the molecular order parameter of a rotationally symmetric fibre (or a grating of the fibres in parallel) we introduce the second Legendre polynomial as a definition of the molecular order parameter ${ }^{19}$

$$
P_{2}(\cos \theta)=\frac{1}{2}\left(3 \cos ^{2}(\theta)-1\right),
$$

where $\theta$ describes the angle between a principle axis $(z$ direction, the fibre axis) and the transition dipole moment vector. In case of rotational symmetry its ensemble average value $\left\langle P_{2}\right\rangle$ is identical with the molecular order parameter $S .{ }^{1}$

$$
\left\langle P_{2}(\cos \theta)\right\rangle=\frac{1}{2}\left(3 \frac{\int_{0}^{\pi} G(\theta, \omega) \cos ^{2}(\theta) \sin (\theta) \mathrm{d} \theta}{\int_{0}^{\pi} G(\theta, \omega) \sin (\theta) \mathrm{d} \theta}-1\right)
$$

Note that averaging $(\langle\ldots\rangle)$ over all illuminated molecules is inherent in IR absorption measurements. Assuming a Gaussian shaped dipole distribution density $G(\theta)$ with its central moment $\theta_{0}$ located at $0^{\circ}$ (as derived from polarization measurements on fibres in parallel $\left.{ }^{5}\right)$ and a certain distribution width $\omega_{0}$,

$$
G(\theta)=\frac{1}{\sqrt{\pi \omega_{0}^{2}}}\left[\exp \left(-\frac{\left(\theta-\theta_{0}^{(1)}\right)^{2}}{2 \omega_{0}^{2}}\right)+\exp \left(-\frac{\left(\theta-\theta_{0}^{(2)}\right)^{2}}{2 \omega_{0}^{2}}\right)\right]
$$

and taking $D=22.4,20.1$, and 17.3 while employing the relation between $S=\left\langle P_{2}(\cos \theta, \omega)\right\rangle$ and $D($ ESI S2 $\dagger$ ) one is able to obtain the width of the microscopic dipole distribution as $\omega_{0}=12.0^{\circ}$, $12.7^{\circ}$, and $13.6^{\circ} .{ }^{1}$ Note that the second term $\left(\theta_{0}^{(2)}=\theta_{0}^{(1)}+180^{\circ}\right)$ results from periodic boundary conditions and has nothing to do with mathematical derivative. Normalization to 1 is maintained when integrating $G(\theta)$ from $\theta=0^{\circ}$ to $180^{\circ}$.

Furthermore, to model the system composed of rotationally symmetric fibres forming a disturbed grating it is useful to describe it through the microscopic distribution of the molecular transition dipole moments within one fibre $G(\theta)$ convolved with the geometrical (macroscopic) distribution of the fibres $\bar{G}(\vartheta, \phi)$. The microscopic dipole distribution is handled as stated in the former paragraph. The geometrical distribution of the fibres is deduced from a micrograph of the fibres' arrangement within the sample compartment as describe above. Additionally, we assume that the grating's deformation, 
and hence geometrical distortion, mainly take place within a plane parallel to the diamonds' surfaces ( $x z$ plane). This is justified, because the grating is in direct contact to one diamond at which it is supported. Due to the deformation, rotational symmetry, as in case of fibres in parallel orientation along the $z$ direction, is not adequate anymore. To handle the asymmetry, $\bar{G}(\vartheta, \phi)$ is separated into two terms: one describes the fibres' orientation within the $x z$ plane and is assigned to $\cos ^{2}(\phi)$ ( $x$ direction), the other is assigned to $\sin ^{2}(\phi)$ and describes the geometry in the $y z$ plane ( $y$ direction). In case there would be no difference between the dipole distributions in the two directions $\left(\bar{G}_{x}=\bar{G}_{y}\right)$ one is able to apply $\sin ^{2}(\phi)+\cos ^{2}(\phi)=1$ with the result that $\bar{G}(\theta, \phi)$ is independent of $\phi$ and the system exhibits rotational symmetry. Because the dipole distribution within the $y z$ plane is much less affected by geometrical distortions than within the $x z$ plane, the former can be treated analogously to the undisturbed case, wherefore $\bar{G}_{y}$ is modelled by a Dirac delta function $\delta(\vartheta)$ resulting in 1 during the convolution process. Thus, we define

$$
\bar{G}(\vartheta, \phi)=\bar{G}_{x}(\vartheta) \cos ^{2}(\phi)+\bar{G}_{y}(\vartheta) \sin ^{2}(\phi)
$$

with

$$
\begin{gathered}
\bar{G}_{x}(\vartheta)=\frac{\tilde{N}\left(\vartheta_{i}\right)}{\sum_{j} \tilde{N}\left(\vartheta_{j}\right)} \delta\left(\vartheta-\vartheta_{i}\right) \\
\bar{G}_{y}(\vartheta)=\delta(\vartheta-0) .
\end{gathered}
$$

$\tilde{N}\left(\vartheta_{i}\right)$ represents the counts of the histogram $N\left(\vartheta_{i}\right)$ according to the fibre segment's direction $\vartheta_{i} \in\left[-30^{\circ},-25^{\circ}, \ldots,+30^{\circ}\right]$ corrected for the angle-dependent sample volume within one square region $\tilde{N}\left(\vartheta_{i}\right)=N\left(\vartheta_{i}\right) / \cos \left(\vartheta_{i}\right)$.

After equating the principle axes of the microscopic (fibre fixed) and macroscopic (geometrical) coordinate systems $(\theta=\vartheta)$ and performing the convolution (ESI S3†) one obtains the dipole distribution density of the geometrical disturbed grating $\tilde{G}(\theta, \phi)$ which allows for the determination the absorbance strength with respect to the principle axes $\left(A_{x x}, A_{y y}\right.$, and $\left.A_{z z}\right)$ and, hence, the dichroic ratio, as well as, order parameter values. ${ }^{1}$

$$
\begin{aligned}
& A_{x x}=C \int_{0}^{2 \pi} \int_{0}^{\pi} \tilde{G}(\theta, \phi) \sin ^{3}(\theta) \cos ^{2}(\phi) \mathrm{d} \theta \mathrm{d} \phi \\
& A_{y y}=C \int_{0}^{2 \pi} \int_{0}^{\pi} \tilde{G}(\theta, \phi) \sin ^{3}(\theta) \sin ^{2}(\phi) \mathrm{d} \theta \mathrm{d} \phi \\
& A_{z z}=C \int_{0}^{2 \pi} \int_{0}^{\pi} \tilde{G}(\theta, \phi) \sin (\theta) \cos ^{2}(\theta) \mathrm{d} \theta \mathrm{d} \phi
\end{aligned}
$$

The parameter $C$ represents measurement-dependent values $\left(C=E_{0}{ }^{2} \mu_{0}{ }^{2} N\right)$ as the electric field strength or the transition dipole moment, as well as a normalization constant $\left(\int_{0}^{2 \pi} \int_{0}^{\pi} \tilde{G}(\theta, \phi) \sin (\theta) \mathrm{d} \theta \mathrm{d} \phi=4 \pi\right)$. When calculating the dichroic ratio within the $x z$ plane,

$$
D_{z x}=\frac{A_{z z}}{A_{x x}}
$$

which is identical to the experimentally determined value $D, C$ is cancelled out. $^{20}$ Using the asymmetric dipole distribution density $\tilde{G}(\theta, \phi)$ deduced from the microscopic description $G\left(\theta \mid \theta_{0}=0^{\circ}, \omega_{0}=13.6^{\circ}\right)$ convolved with the fibre orientation $\bar{G}(\vartheta, \phi)$ as derived form the micrograph and the histogram, the apparent dichroic ratio can be obtained as $D_{z x}=7.2$. It is evident, that this value is higher than the ratio found in case of the spectral measurements indicating a less oriented sample as presumed. Alternatively, using the fitted Gaussian function results in $D_{z x}=4.5$ underestimating the fibres alignment, as already evident in the not-matching wings of the distribution function (Fig. 2 and ESI S4 $\dagger$ ).

So far, we have demonstrated that the spectral dichroism as derived from IR measurements can be treated as convolution of the microscopic transition dipole distribution within a single fibre and the macroscopic, geometrical distribution of the fibres inside the DAC in fair agreement (20\% accuracy) to the measured values. The outcome of the IR experiment depends on both, the microscopic and the macroscopic distribution, whereas optical microscopy can only be used to determine the macroscopic one. By comparing the IR and microscopy measurements, information of the microscopic distribution can be obtained. Hereafter we discuss possible factors that can influence the deviation of $D$ and $D_{z x}$ and derive the change of the microscopic transition moment distribution in dependence on hydrostatic pressure.

Associated with recalculating the dichroic ratio we try to correct the measured dichroism. For that purpose, we fix the geometrical distribution $\bar{G}(\vartheta, \phi)$ while comparing the dichroic ratio depending on a variable microscopic distribution width $\omega_{0}$ with the measured values. Despite the integration during calculating the absorbance values (eqn (9)-(11)) is limiting the determination of an analytical inverse function, the dichroic ratio depending on the expectation $\theta_{0}$ and the width $\omega_{0}$ of the underlying microscopic distribution shows a strict monotonic dependence. Thus, it is handled as an injective transformation and an inverse function exists on a confined region, even when not expressible analytically. ${ }^{21}$ Hence, parameterization of this dependence allows for backward determination. Taking $\omega_{0}=$ $18.1^{\circ}$ a value of $D=5.5$ similar to the on deduced from the spectral measurements is achieved (Fig. 3f). In the former it is shown, that closing the DAC while misaligning the grating at ambient pressure reduces the dichroic ration to a greater extend than the application of hydrostatic pressure afterwards. Thus, an increase of the microscopic distribution width of $33 \%$ of the value of the undisturbed cases is not reasonable. That is why, we believe the origin of the deviation between the calculated and the measured values lies solely in geometry.

Basing on the reasons that are reduce the dichroic ratio we investigate the influence of the fibre orientation. For that purpose, we introduce a model function representing the fibres' (mis-)alignment within the $x z$ plane by replacing $\bar{G}_{x}$ (eqn (7)) with 
$\bar{G}_{x}(\vartheta)=\frac{1}{\sqrt{2 \pi \omega_{1}^{2}}}\left[\exp \left(-\frac{\left(\vartheta-\vartheta_{1}^{(1)}\right)^{2}}{2 \omega_{1}^{2}}\right)+\exp \left(-\frac{\left(\vartheta-\vartheta_{1}^{(2)}\right)^{2}}{2 \omega_{1}^{2}}\right)\right]$

while assuming $\vartheta_{1}=0^{\circ}$. When determining the dichroic ratio depending on the distribution width $\omega_{1}$ it appears that the value $D_{z x}$ corresponds to a width of $\omega_{1}=15.3^{\circ}$, whereas the experimentally derived value $(D \pm 20 \%)$ is in accordance with $\omega_{1}=$ $(18.9 \pm 3.3)^{\circ}$. It seems that the geometrical distribution as derived from the micrograph is underestimating the gratings distortion. On the one hand, fibres beyond the focal plane of the camera can contribute to the IR measurement while they are not visible in the image. On the other hand, the visual evaluation of the geometry depends on the image contrast. Regions with fibres in parallel, as well as, highly unordered regions are underestimated, due to a lack of contrast, whereas slightly unordered structure are clearly visible.

Assuming a geometrical distribution as described by eqn (6), (8) and (13) and using $\omega_{0}=13.6^{\circ}$ and $\omega_{1}=18.9^{\circ}$ one obtains a dichroic ratio of $D=5.5$, in accordance with our measurements, and an apparent order parameter of $S=0.68$ (eqn S1 $\dagger$ ). For comparison, the application of eqn $\mathrm{S} 2 \uparrow$ would result in $S=0.60$ clearly underestimating the ordering of the sample, because the separation between $x$ and $y$ direction is not done.

Taking the parameters $a_{\mathrm{A}}$ and $b_{\mathrm{A}}$ as derived from fitting a linear dependence on the apparent dichroic ratio under hydrostatic pressure (Fig. 3g) we are able to correlate the varying dichroic ratio during pressure application with an altered microscopic dipole distribution width. Note that for estimating the parameters of Table 2 we assume a preserved geometry during pressing and relaxing runs, as indicated by micrographs, and a linear dependence between $D$ and $S$ which, in fact, is not the case over the hole range of possible values, but applicable for the limited range of this experiment.

The clear distinction between the microscopic, rotationally symmetric transition dipole moment distribution density of a single (spider silk) fibre and the asymmetric geometric distribution of the fibres within the DAC allows for correcting the apparent (molecular) order parameter. Assuming a negligible misalignment of the grating within the $y z$ plane (perpendicular

Table 1 Parameters as derived from fitting a sum of two Gaussian functions onto the histogram of Fig. 2, where $v$ represents the mean value, $\omega$ the standard deviation, and $B$ a scaling factor

\begin{tabular}{rrrrrr}
\hline$\vartheta_{1}$ & $-22.7^{\circ}$ & $\omega_{1}$ & $14.4^{\circ}$ & $B_{1}$ & 8434 \\
$\vartheta_{2}$ & $15.7^{\circ}$ & $\omega_{2}$ & $7.7^{\circ}$ & $B_{2}$ & 4070
\end{tabular}

Table 2 Parameters as derived from fitting a linear dependence on the apparent dichroic ratio and deduced distribution parameters

\begin{tabular}{llll}
\hline$D$ & $\omega_{0}$ & $\omega_{1}$ & $S$ \\
\hline 3.74 & $13.6^{\circ}$ & $24.7^{\circ}$ & 0.59 \\
3.52 & $16.0^{\circ}$ & $24.7^{\circ}$ & 0.56 \\
$\Delta \omega_{0}=0.8^{\circ} \mathrm{GPa}^{-1}$ & & $\Delta S=0.01 \mathrm{GPa}^{-1}$ &
\end{tabular}

to the diamonds' surfaces) compared to the deformation within the $x z$ plane we are able to calculate the value of the molecular order parameter without oversimplifying assumptions, for instance macroscopic rotational symmetry. Although the preserved orientation of the fibres in $y$ direction (along the optical axis) in the current study is well justified, it is still an assumption. Remedy could be provided, for example, by staining the fibres fluorescently and following their trace employing a laser scanning confocal microscope.

\section{Conclusion}

A method and its experimental realization to carry out pressure dependent measurements on the molecular order parameter of highly anisotropic samples, as fibres of spider silk for instance, is described. When applying hydrostatic pressure the sample in the Diamond Anvil Cell (DAC) becomes unavoidably misaligned. However, through employing image analysis and an appropriate mathematical formalism, the macroscopic disorder of the sample is quantitatively determined. After correcting for the background signal of the pressure transmitting medium (in this study paraffin oil), the pressure dependence of the IR dichroism of a specific absorption band (polyalanine vibration at $965 \mathrm{~cm}^{-1}$ ) is deduced.

In summary, order and disorder at macroscopic and microscopic scales are separated enabling to determine the pressure dependence of the molecular order parameter of the alanine moieties being located within the nano-crystalline building blocks of spider silk. The response of the molecular order parameter to hydrostatic pressure up to a value of $3 \mathrm{GPa}$ is obtained as $0.01 \mathrm{GPa}^{-1}$.

\section{Acknowledgements}

The authors would like to thank Wycliffe Kipnusu, Emanuel Mapesa, and Thomas Michael for their helpful advices on language and mathematics. Financial support by the Deutsche Forschungsgemeinschaft (DFG) Project B5 within SFB/TRR 102 "Polymers under multiple constraints: restricted and controlled molecular order and mobility" and through the Leipzig School of Natural Sciences "Building with Molecules and NanoObjects" (BuildMoNa) is highly acknowledged.

\section{References}

1 Vibrational Spectroscopy of Polymers: Principles and Practice, ed. P. R. G. Neil, J. Everall and J. M. Chalmers, John Wiley \& Sons Ltd., 2007.

2 M. V. Kakade, S. Givens, K. Gardner, K. H. Lee, D. B. Chase and J. F. Rabolt, J. Am. Chem. Soc., 2007, 129, 2777-2782.

3 V. Fasano, M. Baroncini, M. Moffa, D. Iandolo, A. Camposeo, A. Credi and D. Pisignano, J. Am. Chem. Soc., 2014, 136, 14245-14254.

4 Polymer Spectroscopy, ed. A. H. Fawcett, John Wiley \& Sons Ltd., 1996.

5 P. Papadopoulos, J. Sölter and F. Kremer, Eur. Phys. J. E: Soft Matter Biol. Phys., 2007, 24, 193-199. 
6 A. M. Anton, W. Kossack, C. Gutsche, R. Figuli (Ene), P. Papadopoulos, J. Ebad-Allah, C. Kuntscher and F. Kremer, Macromolecules, 2013, 46, 4919-4923.

7 A. Jayaraman, Rev. Mod. Phys., 1983, 55, 65-108.

8 A. Jayaraman, Rev. Sci. Instrum., 1986, 57, 1013-1031.

9 D. J. Dunstan and I. L. Spain, J. Phys. E: Sci. Instrum., 1989, 22, 913.

10 I. L. Spain and D. J. Dunstan, J. Phys. E: Sci. Instrum., 1989, 22, 923.

11 P. Colomban, G. Sagon, M. Lesage and J. M. H. Ramirez, Vib. Spectrosc., 2005, 37, 83-90.

12 R. Ene, C. Krywka, S.-G. Kang, P. Papadopoulos, M. Burghammer, E. D. Cola, M. Müller and F. Kremer, Polymer, 2012, 53, 5507-5512.

13 C. Krywka, C. Sternemann, M. Paulus, M. Tolan, C. Royer and R. Winter, ChemPhysChem, 2008, 9, 2809-2815.
14 J. W. Otto, J. K. Vassiliou and G. Frommeyer, Phys. Rev. B: Condens. Matter Mater. Phys., 1998, 57, 3253-3263.

15 K. Syassen, High Pressure Res., 2008, 28, 75-126.

16 R. Ene, P. Papadopoulos and F. Kremer, Soft Matter, 2009, 5, 4568-4574.

17 D. J. Dunstan, Rev. Sci. Instrum., 1989, 60, 3789-3795.

18 A. L. Stancik and E. B. Brauns, Vib. Spectrosc., 2008, 47, 6669.

19 M. Warner and E. M. Terentjev, Liquid Crystal Elastomers, Oxford University Press, 2003.

$20 \mathrm{~J}$. Michl and E. W. Thulstrup, Spectroscopy with polarized light, VHC Publishers, Inc., 1995.

21 Taschenbuch der Mathematik, ed. I. N. Bronstein, K. A. Semendjajew, G. Musiol and H. Mühlig, Verlag Harri Deutsch, Thun and Frankfurt (Main), 2001. 